\title{
Robot-Assisted Laparoscopy, Natural Orifice Transluminal Endoscopy, and Single-Site Laparoscopy in Reproductive Surgery
}

\author{
Antonio R. Gargiulo, M.D., ${ }^{1}$ and Ceana Nezhat, M.D. ${ }^{2}$
}

Minimally invasive gynecologic surgery is continuously pushing its limits by embracing ever more sophisticated technology. This is also true for reproductive surgery, arguably the birthplace of gynecologic endoscopy, where minimally invasive treatment of uterine, tubal, ovarian, and peritoneal pathology has long become the gold standard. This article describes in some detail three novel minimally invasive surgery approaches that have seen the light during the past decade: robot-assisted laparoscopic surgery, natural orifice transluminal endoscopic surgery, and single-incision laparoscopic surgery. These fascinating technologies, far from being widely adopted, are sure to generate scientific controversy for years to come. Nonetheless, they follow in the footsteps of the tradition of innovation that is a defining aspect of our specialty and hold the promise to potentially revolutionize the field of reproductive surgery.

KEYWORDS: Robotic surgery, robotics, natural orifice transluminal endoscopic surgery, NOTES, single-incision laparoscopic surgery, SILS, laparoscopic single-site surgery, LESS, reproductive surgery

Just when advanced laparoscopic technique has become more standardized and the laparoscopic approach is finally being embraced even outside of the minimally invasive "sanctuary" of reproductive surgery, radically new techniques are being introduced that spark familiar controversies and shatter surgical dogmas all over again. Robot-assisted surgery brings stereoscopic vision and intuitive instrument control back to laparoscopy, natural orifice transluminal endoscopy eliminates incisions of the skin and fascia, and single-incision laparoscopy aims at limiting these points of entry. The first technique proposes to bridge the technical gap between open surgery and laparoscopy, and the other two push the limits of minimal invasiveness at the cost of further raising the bar of the technical skills required. Together or separately, sooner or later, these techniques are likely to impact the way we will perform reproductive surgery in the future.

\section{ROBOT-ASSISTED REPRODUCTIVE SURGERY}

Reproductive surgeons abide by the principles of microsurgery. Laparoscopy represents the natural evolution of classic microsurgery: its closed approach limits peritoneal trauma and promotes hemostasis. It also provides tissue
${ }^{1}$ Center for Infertility and Reproductive Surgery, Brigham and Women's Hospital, and Department of Gynecology and Reproductive Biology, Harvard Medical School, at Boston, Massachusetts; ${ }^{2}$ Department of Obstetrics and Gynecology at Northside Hospital, Emory University School of Medicine, and Stanford University School of Medicine, Atlanta, Georgia.

Address for correspondence and reprint requests: Antonio $\mathrm{R}$. Gargiulo, M.D., 75 Francis Street, Boston, MA 02115 (e-mail: agargiulo@partners.org).
The Role of Modern Reproductive Surgery for the Evaluation, Therapy, and Preservation of Fertility; Guest Editor, Keith Isaacson, M.D.

Semin Reprod Med 2011;29:155-168. Copyright (C) 2011 by Thieme Medical Publishers, Inc., 333 Seventh Avenue, New York, NY 10001, USA. Tel: +1(212) 584-4662.

DOI: http://dx.doi.org/10.1055/s-0031-1272478.

ISSN 1526-8004. 
magnification and lighting conditions comparable with those achieved in microsurgery. Thanks to improved patient acceptability and a lower rate of complications, laparoscopy has replaced almost all open procedures in the reproductive specialist's armamentarium. ${ }^{1,2}$

This has come at a price. Laparoscopy has inherent sensory and mechanical limitations compared with open surgery. Sensory limitations include loss of stereoscopic vision and partial loss of tactile sensation. Mechanical limitations are caused by operating through a fulcrum (the anterior abdominal wall) with levers (the shafts of the laparoscopic instruments). In open surgery, the surgeon's upper limbs handle instruments with seven degrees of freedom: the elbow provides yaw (left-right movement about the transverse axis), pitch (up-down movement about the vertical axis) and insertion (in-out movement), the wrist provides another level of yaw and pitch as well as providing roll (rotation around the longitudinal axis); finally the hand provides grip (openclose movement). In laparoscopy we lose yaw and pitch at the wrist. Lack of these movements is particularly taxing during microsurgical procedures because we are used to performing our fine yaw and pitch movements with our wrists, rather than our elbows.

Working through a fulcrum also establishes a counterintuitive working environment where every yaw and pitch of an instrument in the pelvis must correspond to a diametrically opposed movement outside of the body. Finally, operating through long instruments allows an amplification of natural muscle tremors that is not ideal for microsurgical applications.

Many excellent gynecologic surgeons who cannot afford the time and effort required to become proficient laparoscopists are faced with a professional dilemma: to persevere in offering conventional surgery or to start a pattern of referral to gynecologists trained in minimally invasive surgery. What has happened to the field of reproductive surgery during the past 2 decades follows the same practice-shift pattern, but the ramifications of such a shift are more complex. Gynecologic surgery in women facing reproductive challenges should be approached with a comprehensive plan fostering their reproductive endeavor. In this perspective, reproductive surgery encompasses virtually every conservative gynecologic operation during the reproductive years. At a very minimum, it includes all those techniques aimed at the restoration of reproductive structures (tubal, uterine, and ovarian surgery) and at the conservative management of pelvic endometriosis.

It would seem that indications for reproductive surgery abound. Yet a contraction of the field of proper reproductive surgery is more apparent than general statistics seem to suggest. ${ }^{3}$ This is due to the fact that many reproductive endocrinology and infertility (REI) subspecialists refer their surgical patients to gynecologists trained in minimally invasive surgery because the operations where they can best contribute their knowledge and understanding of the field have become too complex to be mastered within an already demanding assisted reproductive technology (ART) practice. This pattern of referral represents, in our view, a concerning disconnection from subspecialty care.

It is in this environment that robotic surgical platforms are coming of age, with a potential to induce a paradigm shift in reproductive surgery.

\section{SURGICAL ROBOTS: A TECHNOLOGY IN RAPID DEVELOPMENT}

At the time in which this article was written there is only one robot being used in gynecologic surgery worldwide and approved for this specific use by the U.S. Food and Drug Administration (FDA): the da Vinci surgical system (Intuitive Surgical, Sunnyvale, CA).

The setup of the da Vinci surgical system is based on the principle of robotic telepresence: The main surgeon is physically removed from the operating table and guides the movements of a passive patient-side robotic device while sitting at a master console. The da Vinci $\mathrm{Si}$ model also supports an assistant surgeon's console. The surgeon operates the master console through two hand controls and several foot pedals. Each of the hand controls is designed to accommodate the surgeon's thumb and opposing finger and allows complete freedom of upper limb movement in three dimensions. These movements are translated and downscaled into movements of the robotic arms at the patientside cart, and into fine movements of the interchangeable "wristed" robotic instruments that the surgeon elects to connect to the robotic arms during each step of any given case (Fig. 1).

The robotic three-dimensional (3D) endoscope enters the abdominal cavity through an $8.5-$ or $12-\mathrm{mm}$ cannula placed at or above the umbilicus (a dedicated camera port has been designed for the new-generation 8.5-mm 3D endoscope). Robotic instruments enter through dedicated 5- or 8-mm steel cannulas. Although $5-\mathrm{mm}$ robotic instruments do exist, their use in gynecologic surgery is limited at this time, mostly due to the lack of electrosurgical instrumentation.

The transposition of the elbows' movements in the transverse axis (yaw) and vertical axis (pitch) is automatically inverted at the level of the robotic arms so the surgeon can perform intuitive movements at the console just as if he or she was operating in a conventional open case. Moreover, the accuracy of movement of the robotic arms and instruments can be scaled down to the surgeon's preference. The surgeon's hand movements at the console occur in a fluid and unrestricted environment. This comes at the expense of tactile sensation (haptic feedback). Simulated haptic feedback is one of the expected improvements of future robotic 


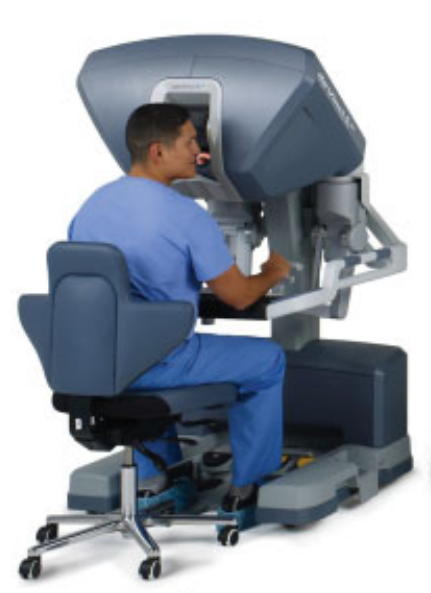

A

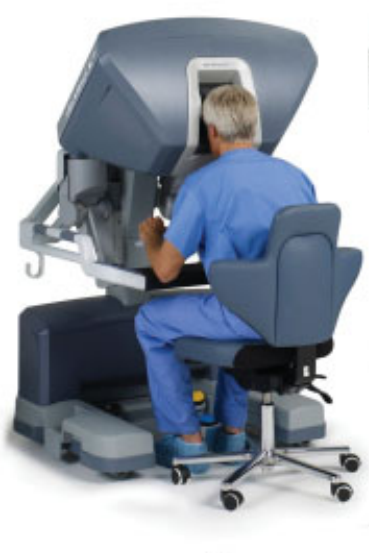

B

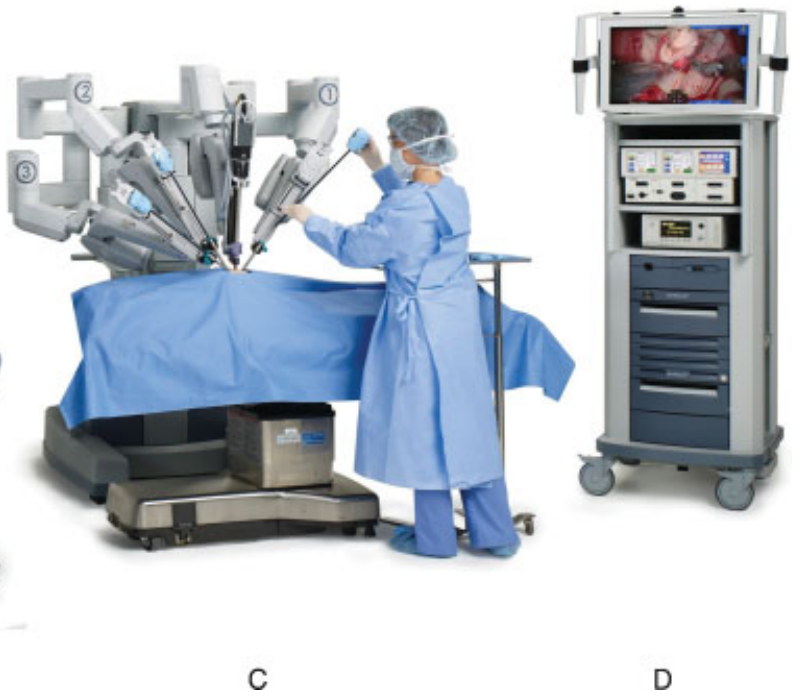

C

Figure 1 Current version of the da Vinci Si robotic surgical platform by Intuitive Surgical. (A) Surgeon's console. (B) Optional second surgeon's console. (C) Patient-side cart with four robotic arms and exchangeable instruments. (D) Laparoscopic tower with main computer. (Copyright Intuitive Surgical, Inc. Reproduced with permission.)

systems. For the time being, surgeons must learn to compensate for the complete loss of tactile sensation with the much improved visual clues allowed by a highdefinition 3D binocular visor.

Laparoscopic surgeons, who are accustomed to depending on elusive foot pedals, quickly learn to appreciate the ergonomics of the da Vinci console's pedal platform. The left side of the pedal platform contains all of the main operational pedals: clutch, instrument switch, and camera motion. The right side of the pedal platform is dedicated to powering the energy sources employed by some of the robotic instruments. The clutch disengages the hand controls of the master console from the robotic arms of the patient-side cart. This allows for continuous optimal positioning of the surgeon's upper limbs during different stages of the operation. The other two left-sided foot pedals are the camera motion pedal (allowing fine control of the robotic arm holding the camera while disengaging all other arms) and the switch allowing alternate use of two of the three robotic instrument arms.

A limitation of the da Vinci surgical system is its size: The massive patient-side cart can make any operating room feel like a small space. This is especially true because lateral docking of the patient-side cart has replaced docking between the patient's legs to improve vaginal access. Therefore miniaturization of surgical robots is one of the first achievements to be expected on the way to a more universal use of these machines. Ideally, robots should be available as more compact units that can be introduced as needed during the flow of any operation, rather than defining the operation as "robotic" from the start. Such a scenario of use ad hoc may sound futuristic because current robotic platforms are quite expensive. In fact, cost seems to be the biggest impediment to the diffusion of this promising technology.

There are several cost analyses in the literature, but only one pertains to gynecologic surgery. This study compared robot-assisted laparoscopic myomectomy with abdominal myomectomy. ${ }^{4}$ The authors matched cases by age, body mass index, and myoma weight. Patients with robot-assisted laparoscopic myomectomy had significantly lower estimated blood loss, complication rate and length of stay when compared with the laparotomy group. Operative times and professional and hospital charges were higher for the robotic group. Professional reimbursement was not significantly different between groups, but hospital reimbursement rates were higher for the robotic. The authors concluded that the costs of robot-assisted myomectomy are higher than those for abdominal myomectomy, but the observed decreased estimated blood loss, complication rate, and length of stay may have a significant societal benefit that will outweigh the upfront financial impact.

Several studies support the argument that robotic surgical platforms are effective technical enablers. One study evaluated the improvement of skill testing before and after an intensive 5-day hands-on minimally invasive surgery training course offered to surgeons. The robotic skill testing scores demonstrated greater improvement than the laparoscopic skill testing scores, suggesting the transfer of laparoscopic skills may be improved using the robotic interface..$^{5}$

Another study compared the quality of suture anastomosis of the ureteropelvic junction obtained with open surgery, conventional laparoscopy and robot-assisted laparoscopy, and it evaluated the surgeons' learning curves. Sutures were performed in 57 pigs by three inexperienced and one experienced surgeon using 
each of the techniques. Operating times were measured. The quality of the anastomoses was evaluated with urodynamic measurements and histology. Data analysis indicated that, among inexperienced surgeons, the efficiency of performing suturing using robot-assisted laparoscopy is operator independent and requires less time to learn compared with conventional laparoscopy. ${ }^{6}$

Finally, in a more recent study, medical students were shown an instructional video and then were tested in intracorporeal suturing on two identical porcine Nissen fundoplication models. ${ }^{7}$ The students were asked to place sutures using conventional laparoscopic instruments in one model and using robotic assistance in the other, in random order. Workload was assessed using the validated National Aeronautics and Space Administration task load index questionnaire, which measures the subjects' self-reported performance, effort, frustration, and the mental, physical, and temporal demands of the task. The study showed that, compared with standard laparoscopy, robotic assistance significantly improved intracorporeal suturing performance and the safety of novices in the operating room while decreasing their workload. Moreover, the robot significantly shortened the learning curve.

No similar studies exist at this time to compare the learning curve of actual laparoscopic and robot-assisted procedures. Studies have been published on the learning curve of certain robot-assisted gynecologic operations. These have arbitrarily defined surgical speed as the main outcome variable. It is expected that a great variability will exist in the learning curve of different types of surgery. The curve is also likely to depend on the baseline laparoscopic and surgical skills of the team, as well as on its surgical volume. Even with these limitations, these studies give us an idea of what is required to master certain robot-assisted techniques. For example, operative times for hysterectomies performed at a general gynecology practice stabilized at $\sim 95$ minutes after 50 cases. ${ }^{8} \mathrm{~A}$ more recent study performed by a gynecologic oncology team to define the learning curve for robotic hysterectomy and pelvic-aortic lymphadenectomy for endometrial carcinoma yielded somewhat different results. ${ }^{9}$ Seventy-nine consecutive patient outcomes were compared between quartiles (cases 1 to 20, 21 to 40, 41 to 60, and 61 to 79 ), and proficiency was defined as the point at which the slope of the curve becomes less steep for operative times. Operative time decreased from the first 20 cases to the next 20 but did not significantly change over the next three quartiles. The authors concluded that proficiency for robotic hysterectomy with pelvic-aortic lymphadenectomy for endometrial cancer is achieved after 20 cases. However, the number of procedures to gain efficiency (i.e., the time when the slope of the curve equals zero) varies for each portion of the case. Finally, in a series of 80 robot-assisted sacrocolpopexies, the mean operative time decreased by $25.4 \%$ after only 10 cases, inducing the authors to conclude that the operation has a short learning curve. ${ }^{10}$

In conclusion, robotic surgical platforms overcome the limitations of conventional laparoscopy, and learning curves for gynecologic operations appear to flatten within the first 50 cases. The high cost of this technology is holding back a more widespread use. Expected market competition should induce an acceleration in the diffusion and advancement of robotic surgery. Future technical improvements, aside from the previously mentioned miniaturization, are likely to include simulated haptic feedback, gaze-based cameras with eye tracker and autofocus, ultra-miniaturization for "wristed" single-port applications, image fusion, and, eventually, active robotic features (such as autonomous knot tying).

\section{ROBOTIC APPLICATIONS IN REPRODUCTIVE SURGERY}

\section{Robot-Assisted Laparoscopic Tubal Reanastomosis}

The evidence for the effectiveness of tubal surgery in the management of infertility is limited, ${ }^{11}$ and robotic surgery is unlikely to make an impact in this field in the era of ART. However, surgery has an important role in the management of regret of tubal sterilization. The first feasibility study for tubal reanastomosis on the da Vinci surgical system was published by Degueldre et al. ${ }^{12}$ Two case series compared robot-assisted tubal reanastomosis performed with the da Vinci surgical system with conventional microsurgical reanastomosis through minilaparotomy. The case-control study by Rodgers et al compared 26 robot-assisted tubal reanastomosis cases with 41 reanastomoses performed by outpatient minilaparotomy. ${ }^{13}$ Surgical times were significantly longer for the robot compared with open surgery. Robotic reanastomosis was also more costly, with a median cost differential of $\$ 1446$ (cost analysis did not include the base cost of the surgical system and the annual maintenance fee). Hospitalization times, pregnancy (61\% robotic versus $79 \%$ minilaparotomy), and ectopic pregnancy rates were not significantly different. Complications occurred less frequently in the robotic group. and the return to normal activity was shorter in this group by $\sim 1$ week. The prospective cohort study by Dharia Patel et al compares 18 robot-assisted tubal reanastomosis cases and 10 open microsurgical tubal reanastomosis cases with hospital admission. ${ }^{14}$ Surgical times were significantly longer for the robot compared with open surgery This group did not perform outpatient minilaparotomy, whereas all patients undergoing robot-assisted surgery were discharged home on the day of surgery. Hence hospitalization times were shorter in the robot-assisted than in the open surgery. Time to 
recovery was significantly less for the robot-assisted reanastomosis group compared with the open surgery group (11.1 days; range: 2 to 28 days, and 28.1 days; range: 21 to 42 days, respectively). Pregnancy (62.5\% robotic versus $50 \%$ open) and ectopic pregnancy rates were not significantly different. The hospital cost for robot-assisted reanastomosis was $\$ 13,773$ (versus $\$ 11,742$ for the open procedure). However, the cost per delivery was similar between the two procedures.

The data seem to indicate that robot-assisted tubal reanastomosis is safe and its results are comparable with those obtained by classic tubal microsurgery performed by trained REI subspecialists. Cost analysis is controversial, but it would appear that even at the current high operating costs, open surgery is cost effective only if patients are sent home within a few hours but not if they stay overnight.

Robotic tubal reanastomosis is performed with either a three- or a four-arm configuration with the assistant port in one of the lower quadrants (Fig. 2) so the exceptionally small needles being passed in and out of the patient can always travel in front of the laparoscope and away from the bowel. As in all robot-assisted reproductive surgery techniques, we prefer lateral docking of the patient-side cart, which allows ample space for access to uterine positioning devices. Typical robotic instrument configurations include a first stage employing ProGrasp forceps, Potts Scissors, and Micro-Bipolar forceps (all Intuitive Surgical) for preparation of the tubal stumps and placing the stent, and a second stage employing ProGrasp forceps and two Black Diamond Micro Forceps (Intuitive Surgical) for suturing. We employ ultrafine (1:5) downscaling on the da Vinci $S$ and fine (1:3) downscaling on the da Vinci Si. Fig. 3 summarizes the technique.

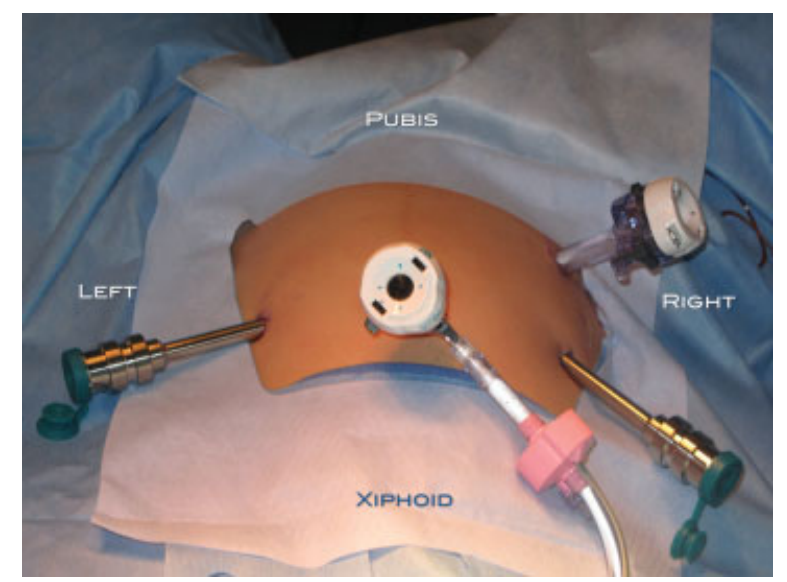

Figure 2 View of lower abdomen with our standard setup for robot-assisted reproductive surgery. The 12-mm primary trocar is placed through an umbilical incision, da Vinci 8-mm trocars are placed 8 to $10 \mathrm{~mm}$ to either side of it with a 15 to 30 degree caudal angle, and a patient-side assistant trocar is placed in the right lower quadrant. (Photo courtesy of $A$. Gargiulo and S. Srouji, Brigham and Women's Hospital.)
Most reproductive surgeons only perform a limited number of tubal reanastomoses per year. A case could be made that the enabling nature of robotic technology makes this a perfect example of an operation that is more safely learned and performed robotically.

\section{Robot-Assisted Myomectomy}

Three prospective randomized trials showed the safety and reproductive benefits of laparoscopic myomectomy over abdominal myomectomy. ${ }^{13-17}$ Moreover, abundant data have accumulated attesting to the extremely rare occurrence of the dreaded uterine rupture in pregnancies following laparoscopic myomectomy. ${ }^{18,19}$

Yet such good scientific evidence has not significantly impacted the 50-year trend of performing myomectomy through an abdominal incision. The fact that abdominal myomectomy still represents the standard of care in most developed countries is not surprising, given the serious technical challenges of this operation. ${ }^{20}$ That is why the pioneering work by Advincula and his team, who had the foresight of applying robotic technology to myomectomy almost a decade ago, represents a true milestone in our field. In 2004, this group published the first feasibility study with data from 35 patients. ${ }^{21}$ These were not small cases: The mean myoma weight was $223.2 \pm 244.1 \mathrm{~g}$ and the mean diameter was $7.9 \pm 3.5 \mathrm{~cm}$. The median myoma number was 1.6 (range: 1 to 5 ). The mean blood loss was $169 \pm 198.7$ $\mathrm{ml}$; the mean operating time was $230.8 \pm 83$ minutes. Patients went home within a day. This study helped pave the way for FDA clearance of the use of the da Vinci surgical system for this and other gynecologic indications in early 2005. Classically trained reproductive surgeons should take much comfort in following the steps of a robot-assisted myomectomy and see that this is an operation of uncompromised precision. Robot-assisted myomectomy is performed with either a three- or fourarm configuration with the assistant trocar in one of the lower quadrants so the many needles passed in and out of the patient can always travel in front of the laparoscope and away from the bowel. There is another good reason to place the bedside assistant's port in one of the lower quadrants. As you will notice in Fig. 2 the right lower quadrant assistant port and the right robotic port end up positioned on the same vertical line. This means that if conventional laparoscopy is needed for any part of the operation, there already is the ideal trocar placement for that, the "ultra-lateral" port position described by Koh and Janik. ${ }^{22}$

Briefly, (1) the myometrium is infiltrated with dilute vasopressin and a transverse incision is performed with robotic Harmonic shears set at maximum power (which has less thermal spread and produces less smoke than any electrical devices); (2) tenaculum forceps are applied and an "onionskin" technique, ${ }^{26}$ is applied with 

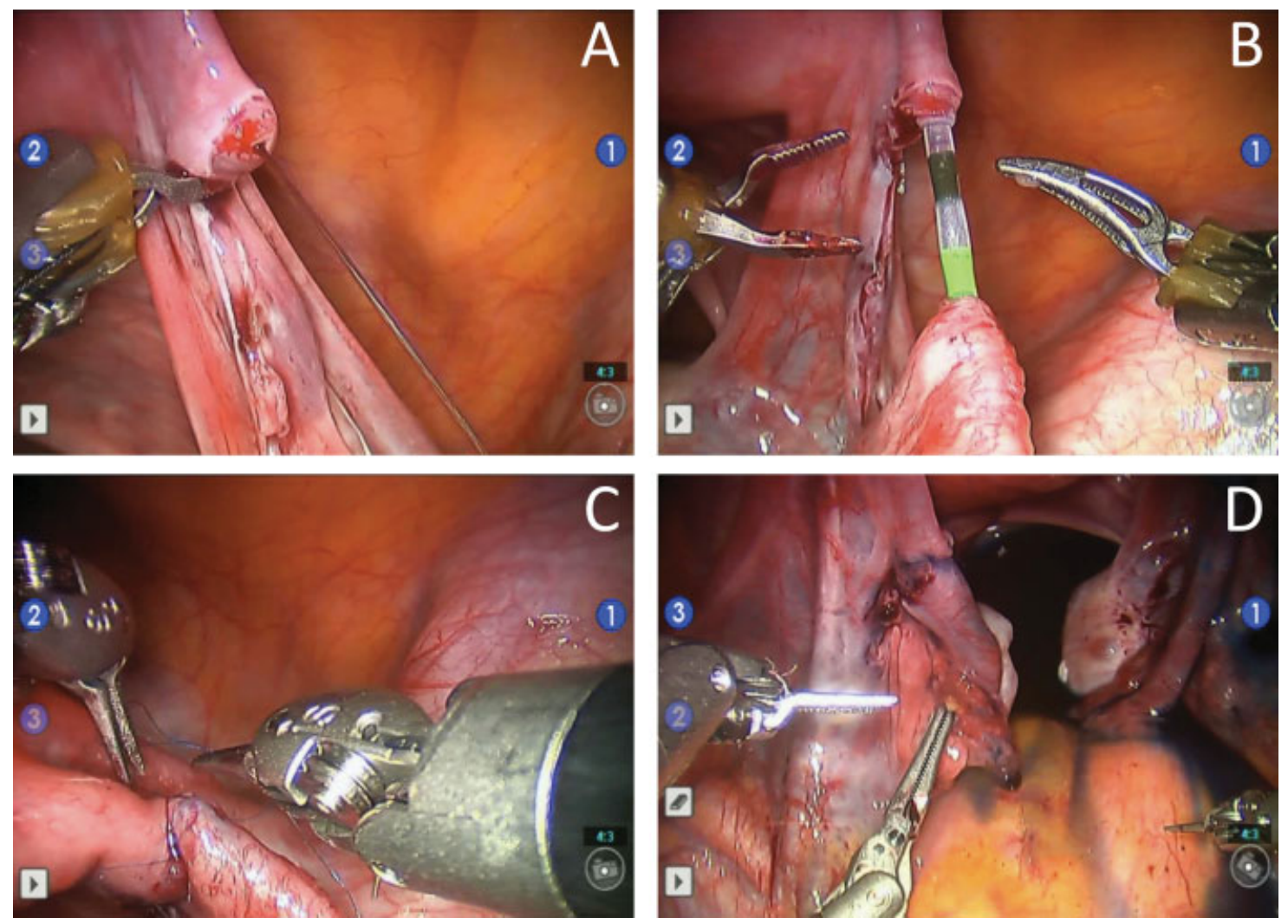

Figure 3 Robot-assisted laparoscopic tubal reanastomosis. (A) Preparation of the proximal stump with flow of dye from the transected tubal lumen. (B) Placement of graduated endoscopic retrograde cholangiopancreatography catheter as stent. (C) Placement of 8-0 polypropylene sutures at 12, 3, 6, and 9 o'clock. (D) Final result with copious bilateral spill at chromotubation. (Photos courtesy of A. Gargiulo and S. Srouji, Brigham and Women's Hospital, Boston, MA.)

robotic Harmonic shears; (3) a Maryland fenestrated grasper is used as a dynamic retractor and to cauterize arteriolar bleeding; (4) the myoma is placed in the posterior cul-de-sac and a myoma count is initiated (if multiple small myomata are removed, they can be kept together on a loop of suture until morcellation); (5) chromotubation is performed after enucleation to identify possible occult endometrial entry; (6) uterine incisions are closed in one to five layers (depending on size and depth) right after each myoma enucleation occurs to minimize blood loss; (7) the endometrium (when entered) is reapproximated with running 3-0 polyglecaprone 25 ; (8) the deep myometrial layer is closed with interrupted figure-of-eight sutures of 0 polyglactin and the outer myometrial layer(s) with running suture(s) of 0 polyglactin; (9) the uterine serosa is closed with $2-0$ or $3-0$ polyglecaprone 25 in a running baseball stitch; (10) extraction of the enucleated myomata from the abdominal cavity is accomplished with an electric morcellator. The technique is summarized in Fig. 4 and Fig. 5.

The recent development of self-anchoring barbed sutures offers a way to decrease operative time in large robot-assisted myomectomies. Successful use of barbed suture in conventional laparoscopic myomectomy has already been described. ${ }^{23}$ We hope evidence of optimal performance of uteri reconstructed with knot-free su- tures (in terms of low chance of rupture) will soon become available. As far as evidence of reproductive safety of robot-assisted myomectomy performed with conventional sutures, it would seem redundant to "reinvent the wheel" after $>50$ years of overall safe obstetric reports following abdominal myomectomy and freehand laparoscopic myomectomy. In any case, tens of women have safely delivered following robot-assisted myomectomy at our institutions, and a large multicenter study addressing this question is currently being prepared for publication. No discussion on robot-assisted myomectomy can be complete without mention of the intense preoperative work to establish stringent indications for the procedure and the most effective operative strategy. The importance of the input of the REI subspecialist on the indications for myomectomy in women facing reproductive challenges cannot be overemphasized. The topic of myomectomy in infertile women is rife with conflicting scientific literature, and important decisions will still have to be made based on extensive professional experience. $^{24}$

Considering that tactile sensation is lost with current robotic platforms, detailed preoperative imaging studies become a fundamental prerequisite for robotassisted laparoscopic myomectomy. Preoperative mapping has several goals: (1) assess the number, size, and 

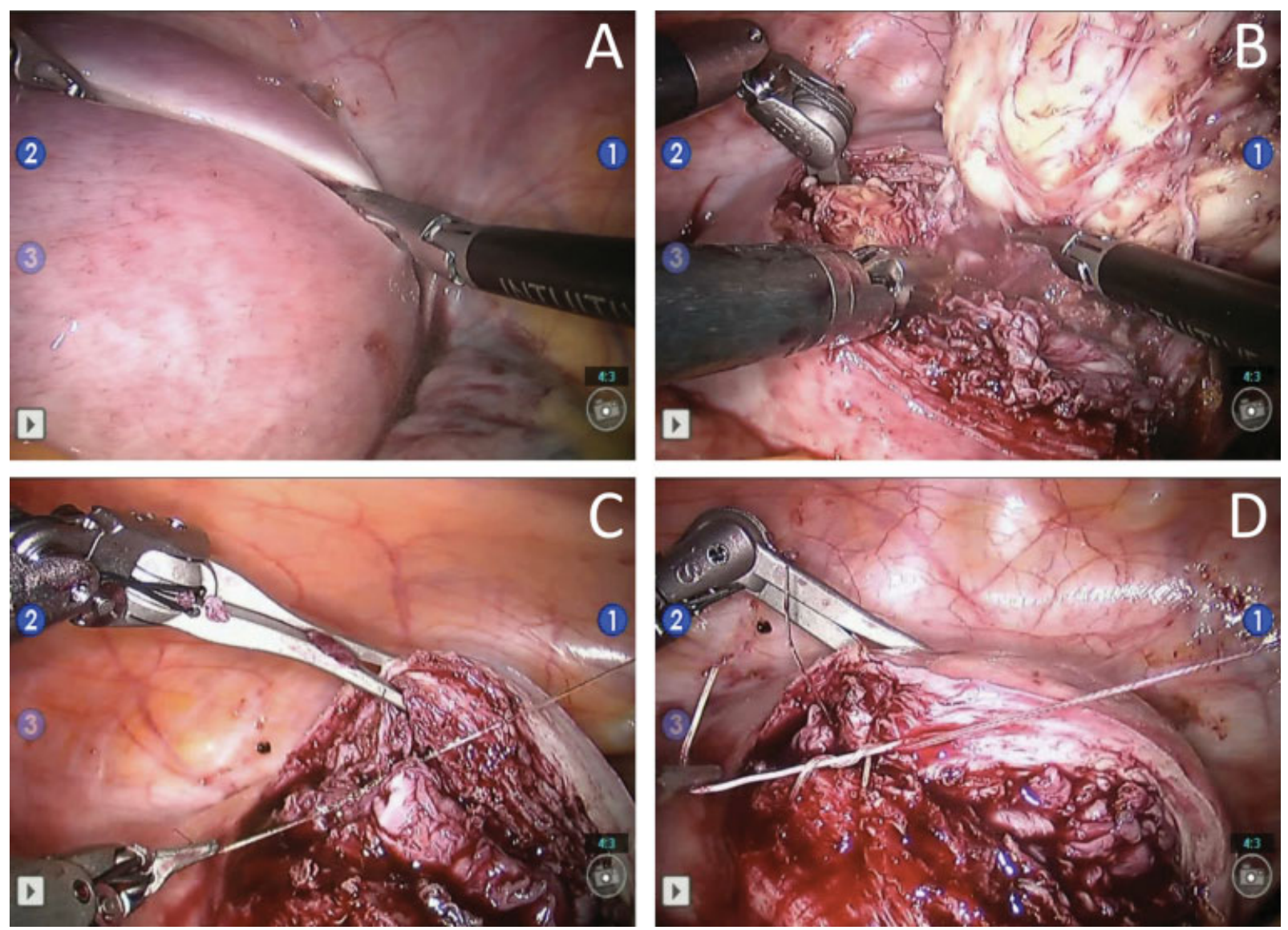

Figure 4 Robot-assisted myomectomy. (A) Incision of the myometrium with robotic harmonic shears. (B) Enucleation of intramural myoma. (C) Reapproximation of the endometrium with 3-0 polyglecaprone 25. (D) Closure of deep myometrial layer with interrupted figure-of-eight sutures of 0 polyglactin. (Photos courtesy of A. Gargiulo and S. Srouji, Brigham and Women's Hospital.)

location of all myomata in reference to the endometrial cavity; (2) rule out adenomyosis; and (3) provide additional reassurance as to the benign nature of large uterine masses. Magnetic resonance imaging (MRI) has a high sensitivity and a low specificity for diagnosing leiomyoma and a high specificity and a low sensitivity for diagnosing adenomyosis, ${ }^{25}$ hence a good transvaginal ultrasound is just as useful as MRI in the mapping of smaller uterine tumors. ${ }^{26}$ In the case of large uterine masses, ultrasound cannot show the type of detailed relationships between the tumor and the uterine cavity that are needed for a safe laparoscopic myomectomy, and MRI with gadolinium enhancement is preferable. Another good reason to prefer MRI in the case of larger uterine masses is that this technique has a better chance of identifying tumors suspected of malignant degeneration. The study by Goto et al showed that the combined use of MRI and serum measurement of lactate dehydrogenase is useful in making a differentiated diagnosis of leiomyosarcoma from nonmalignant degenerated leiomyoma before surgical treatment. ${ }^{27}$ Defining a mass at risk of being a sarcoma is a fundamental step when morcellation is required for tumor extraction. Preoper- ative identification of diffuse adenomyosis precludes effective surgical treatment of any kind. Adenomyomas instead are discrete uterine masses that resemble myomata but have a poorly demarcated plane that typically involves the endometrium. Even so, in our experience these tumors are amenable to satisfactory enucleation with robot assistance.

Two recent studies have compared robot-assisted myomectomy with freehand laparoscopic myomectomy. ${ }^{28,29}$ Both studies were relatively small and, more importantly, compared the proficiency of very advanced conventional laparoscopic teams with robotic teams within the initial learning curve (i.e., $<50$ cases). Not surprisingly, operative times were significantly lower for the conventional laparoscopy cases. However, the entire debate of freehand laparoscopic versus robot-assisted laparoscopic has limited clinical significance in our view. Robot-assisted surgery is just another way to do laparoscopic surgery. Some of us will argue that the quality of the microsurgical and reconstructive work performed robotically is more in keeping with the principles of our specialty, but that has to be demonstrated on a case-by-case basis. The bottom line is that 

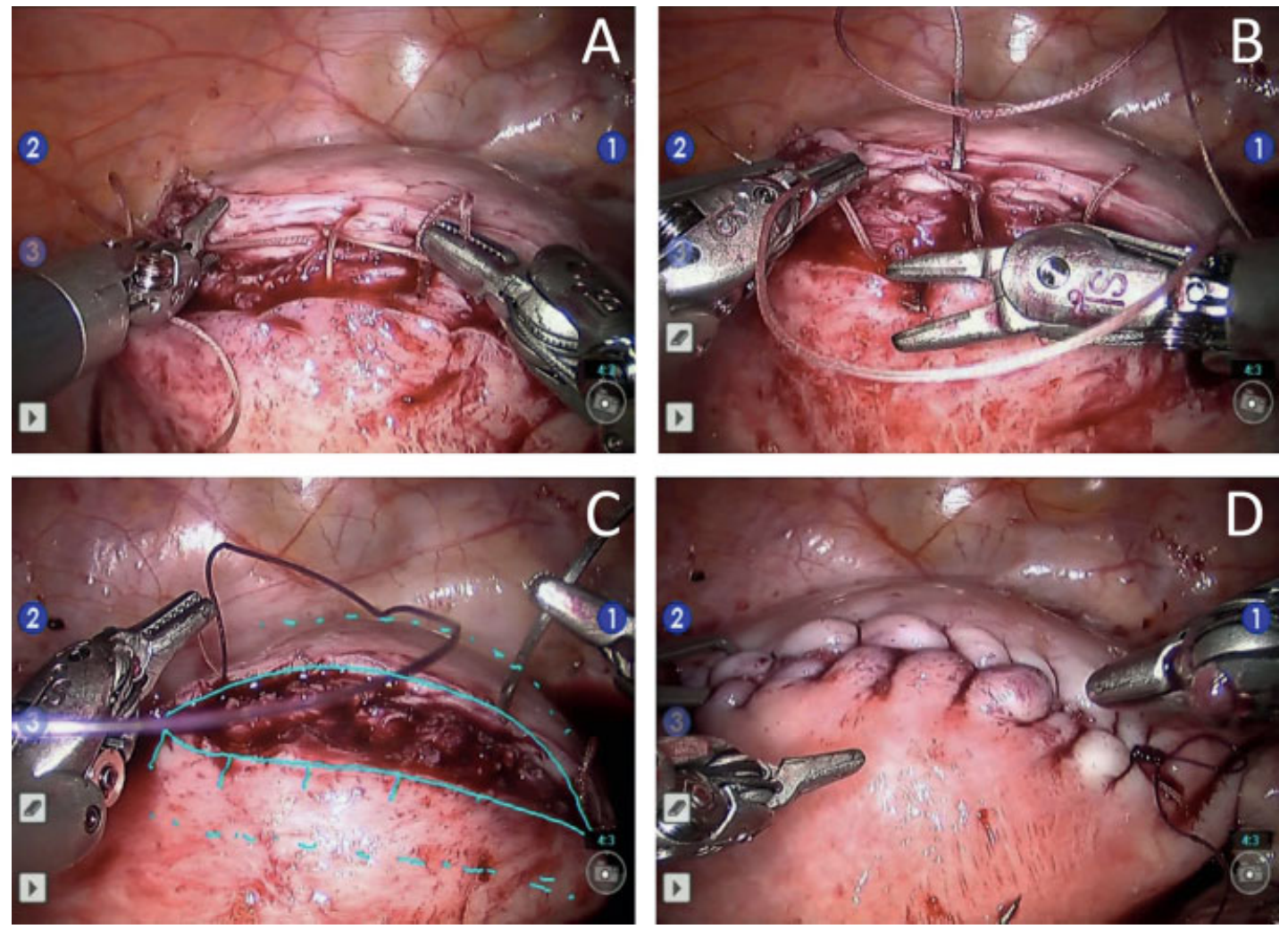

Figure 5 Robot-assisted myomectomy. (A) Completed closure of deep layers. (B) Running suture of 0 polyglactin to close outer layer of myometrium. (C) use of Telestration (USAOPOLY Inc., Carlsbad, CA) to instruct a training robotic surgeon. (D) Closure of the uterine serosa with running baseball stitch of 2-0 polyglecaprone 25. (Photos courtesy of A. Gargiulo and S. Srouji, Brigham and Women's Hospital, Boston, MA.)

current robotic surgical platforms can enable good laparoscopic surgeons to expand their field of action to match that of advanced laparoscopic surgeons. In conclusion, robot-assisted laparoscopic myomectomy allows transposition of the classic abdominal myomectomy technique to the laparoscopic arena. It is safe and reproducible and-with the expected advancements of surgical robotics- has the potential to be adopted by more infertility specialists as part of their armamentarium for comprehensive reproductive care.

\section{Robot-Assisted Debulking of Pelvic Endometriosis}

Endometriosis is yet another clinical scenario in which the decision of how, when, and to what extent to proceed with debulking of the disease should not lie outside of a concerted treatment strategy formulated by an REI subspecialist. ${ }^{30,31}$ However, endometriosis can present some of the most challenging and intimidating surgical scenarios that a gynecologist will ever encounter. Hence the temptation for the reproductive specialist to refer these patients to minimally invasive gynecologic surgeons can be strong. Can the robot come to the rescue in this case and become the enabler it has shown to be for tubal and uterine surgery? Recently, Nezhat et $\mathrm{al}^{32}$ reported on the safe use of the 5-mm da Vinci system to treat endometriosis (Fig. 6). The full report is in process, but while we await publication of case series on robot-assisted debulking of endometriosis, we are glad to share some thoughts derived from our own robotic practices. There is no question that the lack of tactile feedback does pose limitations to the application of current robotic technology to endometriosis. However, exceptional visual feedback and the ability to operate effortlessly in the posterior cul-de-sac provide a rational basis to consider robotic assistance in some case of severe endometriosis. This is, in our view, the most challenging of robot-assisted reproductive surgeries and should be approached only when compensatory visual feedback is well developed and use of the machine has become second nature. These cases are usually long and not particularly hemostatic, and anatomical planes are elusive at best; therefore unhindered concentration is fundamental. In situations like these, another improvement offered by robot-assisted surgery is its advanced ergonomics.

Surgical ergonomics has evolved as a scientific field in parallel with the introduction of complex technology in the operating room. ${ }^{33}$ Its underlying principle 


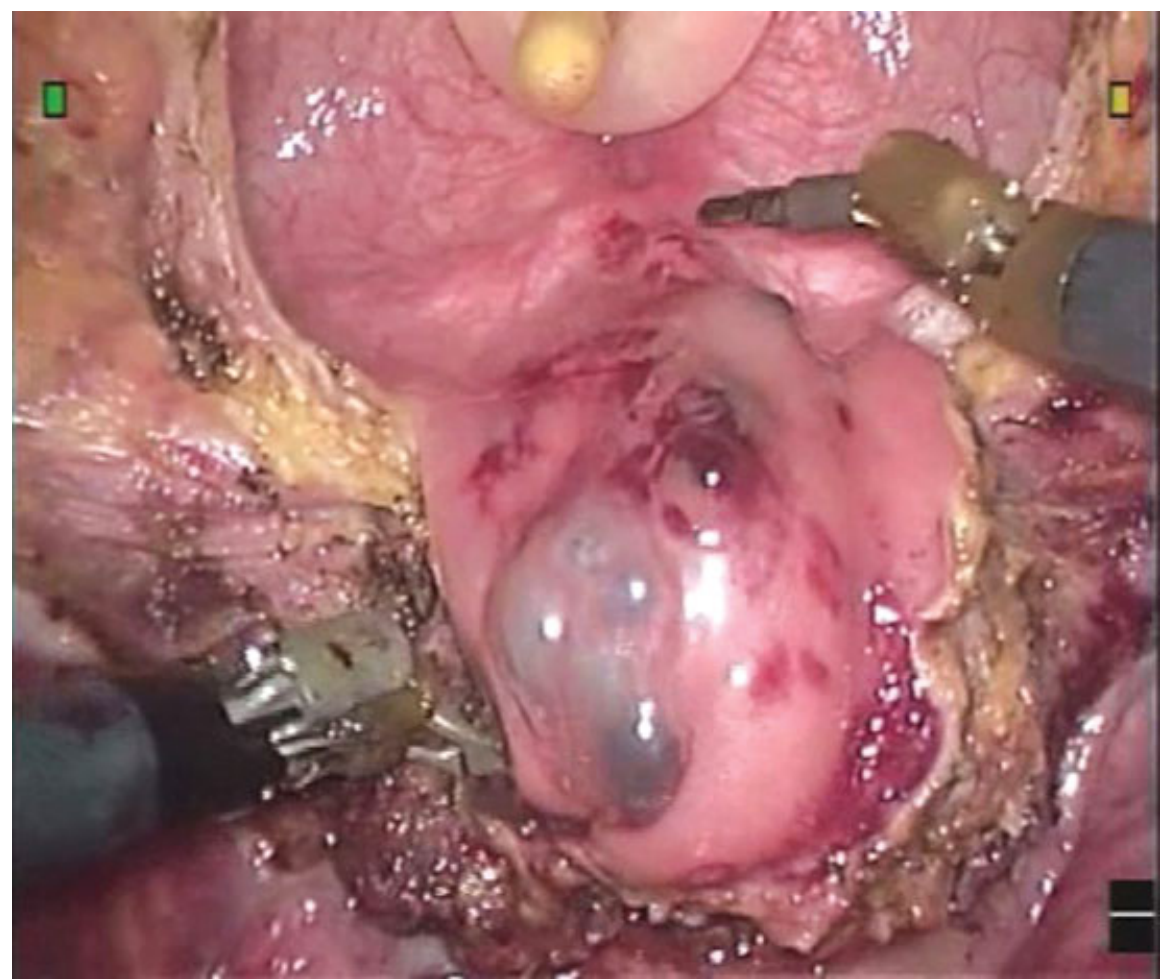

Figure 6 Robotic excision of infiltrating bladder endometriosis. (Photo courtesy of C. Nezhat, Atlanta Center for Minimally Invasive Surgery and Reproductive Medicine.)

is that disruptions to the surgical workflow have been correlated with an increase in surgical errors and suboptimal outcomes in patient safety measures ${ }^{34}$ and therefore must be avoided. Conventional laparoscopic surgery occurs in an operating room environment where disruptions to the workflow abound. For example, we are well aware that gaze disruptions (the surgeon looking away from the laparoscopic screen) occur frequently due to instrument exchange, extracorporeal work, equipment troubleshooting, and communication. Still, how frequently gaze disruptions actually occur may be a little surprising: On average, 40 breaks occurred in the main operating surgeon's attention per 15 minutes of operating time during routine laparoscopic cholecystectomy. ${ }^{35}$ Because robotic surgeons work in an immersive environment, one could safely extrapolate that the amount of gaze disruption in robotic surgery approaches zero. And what about surgeon's generalized fatigue? The data on the negative effects of laparoscopic surgery on the musculoskeletal system of surgeons are nothing short of alarming. Park and colleagues polled $>300$ general surgery laparoscopic specialists in North America and presented evidence that $87 \%$ of them suffer from musculoskeletal occupational injury. ${ }^{36}$ Surgical assistants are not immune to this type of occupational hazard either, as demonstrated by a separate study from the same group. ${ }^{37}$

Because they eliminate the standing and unbalanced posture of surgeons and assistants, as well as the neck strain and the heavy work normally performed by the shoulders, robotic platforms appear to be an effective way to improve the ergonomics of our operating rooms.

In conclusion, recently published data describe the safe use of robot-assisted laparoscopic surgery in endometriosis. Improved visual feedback, instrumentation, and ergonomics seem to compensate for the current absence of haptic feedback and may represent significant advantages over conventional laparoscopy when approaching complex pelvic dissection.

\section{NATURAL ORIFICE TRANSLUMINAL ENDOSCOPIC SURGERY AND SINGLE-SITE LAPAROSCOPIC SURGERY}

For centuries, surgeons have been searching for ways to improve operative outcomes with minimal intervention. The recent advances in laparoscopic techniques have resulted in shorter recovery times, less morbidity, and better cosmetic outcomes. Although we have achieved enormous success in the field of minimally invasive surgery, the quest for perfection and minimal intervention remains as compelling as before. Two of the newest concepts in minimally invasive surgery, natural orifice transluminal endoscopic surgery (NOTES) and singleincision laparoscopic surgery (SILS), also known as laparoendoscopic single-site surgery (LESS), have been on the frontline of innovation and are showing promising results. 


\section{Natural Orifice Transluminal Endoscopic Surgery}

In the field of gynecology, vaginal approaches to surgery have always been a very popular and preferred method. Vaginal hysterectomies are rapidly increasing in popularity due to improved postoperative pain, shorter hospital stays and surgery times, better cosmetic results, and similar outcomes. As part of ART, transvaginal oocyte retrieval under ultrasound guidance has been the standard of care for quite some time for in vitro fertilization. Surgeons naturally began to investigate other procedures that could be done through the vagina and other natural orifices.

NOTES was first proposed in the early 1990s. It is a new form of minimally invasive surgery that is quickly moving from feasibility studies to actual practice. The intent of this approach is to perform surgery through the body's natural orifices (i.e., mouth, vagina, anus) to minimize incisions and disruption of the abdominal or pelvic muscles and fascia. Multiple attempts have been documented, including transcolonic, transgastric, transurethral, and transvaginal approaches. The hope is that this will decrease recovery time and surgical site complications such as infections and hernias, and it will ultimately provide an added cosmetic benefit of no visible incisional scars. ${ }^{38}$

Multiple pilot studies have been published in the last decade addressing the feasibility of this novel technique. The first reports of surgical outcomes were in the early 2000s by Kalloo and colleagues. ${ }^{37}$ Transgastric peritoneoscopy was successfully performed on $1750-\mathrm{kg}$ pigs, demonstrating the technique was technically possible and worth further investigation. All of the pigs recovered from the procedure and were able to tolerate oral intake without adverse events. ${ }^{39}$

In response to this and other successful studies, a committee was formed by the American Society for Gastrointestinal Surgery and the Society of American Gastrointestinal and Endoscopic Surgeons to review the current literature and future possibilities of NOTES. Their conclusion was that animal models had shown promise and human studies were warranted. ${ }^{40}$

A pilot study compared diagnostic laparoscopy with transgastric peritoneoscopy in human subjects with pancreatic masses. In 9 of the 10 patients, the findings correlated between the two techniques. No operative complications were encountered. The conclusion was made that NOTES was feasible and could eventually be at least comparable with traditional laparoscopy. ${ }^{41}$

Steele and colleagues reported a feasibility study that included three patients undergoing laparoscopic gastric bypass surgery. During the surgery, a liver biopsy was performed using a flexible endoscope that was passed through the existing gastrotomy. The biopsies were easily obtained, and the abdomen was explored without any reported difficulty. ${ }^{42}$
One of the first published human series of NOTES involved nine transvaginal cholecystectomies, one transvaginal appendectomy, and one transgastric appendectomy. These were not "pure" NOTES procedures: Eight of the eleven trials were aided by a transumbilical cannula, two had two transabdominal cannulas, and the transgastric appendectomy was aided by two $2-\mathrm{mm}$ abdominal ports. There were no reported postoperative or intraoperative complications in any of the procedures, all patients were sent home by postoperative day 2 , and postoperative pain was reported as minimal, confirming the feasibility of this approach. Three additional patients were enrolled in this study, but NOTES was not performed after visualization of the peritoneal cavity revealed adhesions and inflammation, thus pointing to the potential limitations of such an approach. ${ }^{38}$ In 2009, Nezhat et $\mathrm{al}^{43}$ reported their study of 42 patients who underwent natural orificeassisted laparoscopic appendectomy at the time of laparoscopic hysterectomy. They reported no intraoperative or major postoperative complications (Fig. 7). As with any new technique, there are limitations to these procedures. The main technical issue reported by surgeons is the limited mobility of available instruments. This is likely due to the fact that the instruments being used are not designed specifically for these procedures. The surgeons performing these trials believed this issue could be alleviated with some instrument modifications. ${ }^{38,40,41}$ There is also a theoretical risk that an increased rate of postoperative wound infections may occur due to the use of nonsterile entry. ${ }^{40,41}$ There have been no reported incidents of postoperative infection in these procedures thus far, although data are limited. The ideal way to close entry sites, particularly at the level of the stomach, also remains unclear.

Another significant limitation is the inability to measure and maintain intra-abdominal pressure accurately during these procedures. Bergström et al reported intra-abdominal pressures in a series of transgastric cholecystectomies and tubal resections in a porcine model. A standard Veress needle technique was used to calculate pressure, and surgeons were asked to report when signs or symptoms of high intra-abdominal pressure were noted. Unacceptably high pressures were noted in all of the procedures, and physicians were unaware of the pressures $>50 \%$ of the time. To address this problem, the same group reported a modified feedback control valve that aided in the monitoring and control of intra-abdominal pressure. ${ }^{44}$

A surgical robot system recently was developed with telecontrol function. This system was successfully used in endoscopic procedure with two hands for teleNOTES. ${ }^{45}$ The advent of robotic surgery, combined with a NOTES approach, can change the concept of limiting factors in this newly developing field. 


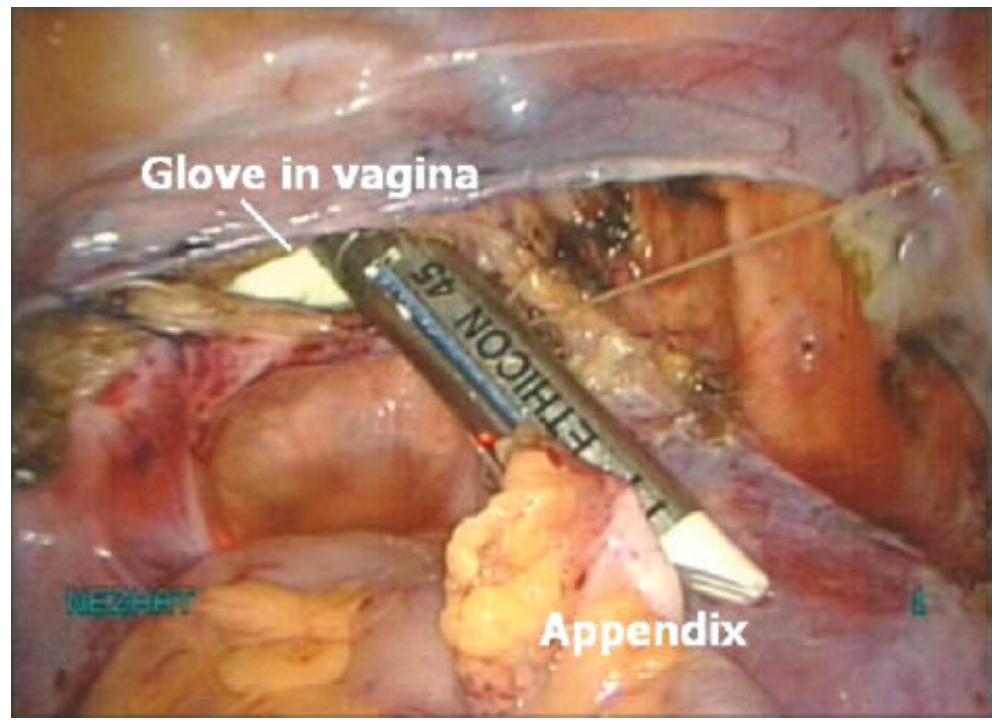

Figure 7 Transvaginal appendectomy. The glove maintains pneumoperitoneum as the stapler and specimen are removed through the colpotomy. (Photo courtesy of C. Nezhat, Atlanta Center for Minimally Invasive Surgery and Reproductive Medicine.)

Although a promising technique, NOTES is still in its infancy. It has shown a great deal of promise with some identifiable limitations that can be modified in future trials. Those limitations and surgeons' perception of the new approach will need to be addressed before the next leap forward of NOTES. Recent survey of practicing gynecologists has demonstrated that although close to $70 \%$ of surgeons think positively about NOTES, $<30 \%$ of physicians would recommend NOTES to their patients. Positive thoughts regarding scarless surgery and quicker recovery times were counterbalanced by concerns for postoperative infection, visceral lesions, infertility, and adhesions. Potential problems such as dyspareunia, infertility, and the spread of preexisting endometriosis were also named as factors in long-term follow up. ${ }^{46}$

\section{Single-Site Laparoscopy}

An additional proposed method of entry for minimally invasive surgery is SILS (or LESS) This technique uses a single, usually umbilical, incision for all instruments rather than multiple port sites as usually used in laparoscopic surgery. The terms are often used interchangeably in the literature, although some authors describe them as two separate techniques. Both techniques are discussed and referred to collectively here as single-site laparoscopy.

Reports of various laparoscopic abdominal surgeries through a single incision first surfaced in the general surgery literature. The most commonly reported procedures are cholecystectomy and gastric banding. Hernandez et $\mathrm{al}^{47}$ published their experience with 100 single-site cholecystectomies that showed promising results. The operating times were similar when compared with conventional laparoscopy controls as were most of the measured outcomes. The authors concluded that single-site laparoscopy is a safe and feasible procedure. ${ }^{45}$ Additionally, a case series in the pediatric population reported similar outcomes between single-site laparoscopy and traditional laparoscopy for splenectomy, cholecystectomy, and appendectomy in all measured end points including postoperative pain. ${ }^{48}$

To address some of the identified problems with loss of pneumoperitoneum and increased stress on fascia, various multiaccess ports have been created, such as the X-Cone (Karl Storz Endoscopy, Tuttlingen, Germany), ASC-Triport (Advanced Surgical Concepts, Bray, Ireland), GelPOINT (Applied Medical, Rancho Santa Margarita, CA), and the SILS Port (Covidien, Mansfield, MA). Each of the devices represents a single port that has three to four canula access sites through which standard laparoscopic instruments are placed. ${ }^{49}$

One small case series of three patients undergoing single-site laparoscopy with a multiaccess port for gastric banding showed improved outcomes and was reported to be technically more feasible and successful in comparison with the same procedure without the multiaccess port. ${ }^{49}$ Another series of 20 single-site laparoscopic cholecystectomies with a single port (R-port, Advanced Surgical Concepts) showed promising results with similar outcomes to laparoscopy. Seventeen of 20 cases were able to be performed through a single site, and postoperative pain was reported as less than with traditional laparoscopy. However, the authors did report a significant amount of difficulty with instrumentation through the single port. ${ }^{50}$

The field of gynecologic surgery was not an exception to this innovative technique. One of the earliest reports of single-site laparoscopy was for treatment of ectopic pregnancy and involved the placement of one 
$10-\mathrm{mm}$ and two $5-\mathrm{mm}$ ports through a single $2.5-\mathrm{cm}$ umbilical skin and $12-\mathrm{mm}$ fascial incision. The authors report that this method can be performed successfully with standard laparoscopic instruments and is therefore attractive and feasible. The patient experienced a significant amount of postoperative pain, which was attributed to pelvic inflammatory disease. ${ }^{51}$

Yoon reported on a series of 20 patients undergoing salpingectomy for the treatment of ectopic pregnancy. All procedures were performed through a single $2-\mathrm{cm}$ vertical umbilical incision without use of any additional ports, including five patients with ruptured ectopic pregnancies, seven with hemoperitoneum, and six with pelvic adhesions. The mean operating time reported was 55 minutes and was showed to decrease with experience. ${ }^{52}$

A series of nine laparoscopic single-site and four robotic single-site gynecologic oncology procedures were reported by Nickles and Escobar. These cases were performed using a multiaccess port (SILS Port Multiple Access Port), which allowed for three laparoscopic instruments to be placed. The port was placed through a 3$\mathrm{cm}$ vertical skin incision made through the umbilicus. There were no reported postoperative complications, surgery and recovery time were at least comparable with laparoscopy if not better, and there was a definite improvement in cosmesis. ${ }^{53}$

As with NOTES and other surgical innovations, implementation of robot-assisted surgery into minimally invasive surgical treatment led to the combination of SILS and robotic technology. Several reports addressed the feasibility of this "hybrid" procedure, including hemicolectomy ${ }^{53}$ and radical prostatectomy, dismembered pyeloplasty, and radical nephrectomy. ${ }^{54}$ Out of the experimentations with single-site laparoscopy and observation of limiting factors, several authors proposed what is known as the "cross-hand technique"; designed to simulate the ipsilateral surgical orientation, it has shown promising results in several trials. ${ }^{55,56}$

Although fascinating and promising, single-site laparoscopy in its current form is far from being adapted into general surgical practice. Several studies reported on incidences of increased postoperative pain, intraoperative complications due to poor visualization, and difficulty maintaining pneumoperitoneum compared with controls. ${ }^{47,57-59}$ Despite these occasional complications, the authors of each series believed that single-site laparoscopic surgery is a technique that warrants further exploration.

As with any evolving technique, some clinical questions have yet to be answered. Long-term data on incisional hernias are not available as of yet. It is possible that with the theoretically increased stress placed on the fascia by multiple instruments, hernia risk may actually be increased. Surgeons report difficulty with visualization using standard laparoscopic instruments, which has led to occasional intraoperative complications. In addition, improved postoperative pain compared with conventional laparoscopy has not been proven, and no reported data on cost effectiveness exist thus far. ${ }^{47,59}$ As with any new procedure, there is a learning curve involved, and many believe the outcomes will improve over time.

In conclusion, both NOTES and single-site laparoscopy are exciting new techniques that have the potential to add to the realm of minimally invasive surgery. However, randomized clinical trials, long-term outcome data, and cost analysis would be necessary before either technique could be adopted into standard clinical practice.

\section{ROBOTIC, NATURAL ORIFICE, AND SINGLE-SITE SURGERY: THE FUTURE OF REPRODUCTIVE SURGERY?}

Surgical robotics are a significant technical enabler that could persuade more REI subspecialists to maintain ownership of their patients' reproductive surgery needs. Its safety and efficacy in tubal and uterine surgery is now well established, and even its role in the surgical management of endometriosis appears promising. Indeed, REI specialists (arguably, the pioneers of modern laparoscopy) have strong fundamentals of endoscopy and therefore represent the ideal substrate for a robotic "revolution." But robotic surgery must first survive what appears to be a turbulent infancy where this technology may be destined to succumb in a radically cost-conscious health-care environment. It appears that the true coming of age of surgical robotics can only happen when the cost of this technology drops significantly.

On the other end of the spectrum are the new technologies of natural orifice transluminal surgery and single-site laparoscopy. Both offer options of ultraminimal invasiveness but at the cost of more technical challenges and limitations than conventional laparoscopy. In a surgical environment that still struggles to embrace traditional laparoscopy, this may seem like a countercurrent move. It does not take much imagination to conclude the future may well lie in a fusion of all of the previously described techniques. Robotic NOTES and robot-assisted single-site laparoscopy just make sense, and early prototypes of this technology are already in use. Upsetting as it may sound to surgeons of our generation, conventional laparoscopy in all of its forms may never have a chance of becoming standard of care. Indeed, there is a chance it may take its place in the history of medicine as an inspiring but anti-ergonomic (and potentially surgeon-crippling) exercise that bridged the span of half a century between the era of open surgery and that of robotic surgery. Time will tell. The dawn of robotics is still an exciting time to be a reproductive surgeon. 


\section{REFERENCES}

1. Isaacson KB. Complications of Gynecologic Endoscopic Surgery. Philadelphia, PA: WB Saunders; 2006

2. Nezhat C, Nezhat F, Nezhat C. Nezhat's Operative Gynecologic Laparoscopy and Hysteroscopy. 3rd ed. New York: Cambridge University Press; 2008

3. Ketefian A, Hu J, Bartolucci AA, Azziz R; Society of Reproductive Surgeons, Inc. Fifteen-year trend in the use of reproductive surgery in women in the United States. Fertil Steril 2009;92(2):727-735

4. Advincula AP, Xu X, Goudeau SIV, Ransom SB. Robotassisted laparoscopic myomectomy versus abdominal myomectomy: a comparison of short-term surgical outcomes and immediate costs. J Minim Invasive Gynecol 2007;14(6):698705

5. Vlaovic PD, Sargent ER, Boker JR, et al. Immediate impact of an intensive one-week laparoscopy training program on laparoscopic skills among postgraduate urologists. JSLS 2008;12(1):1-8

6. Passerotti CC, Passerotti AM, Dall'Oglio MF, et al. Comparing the quality of the suture anastomosis and the learning curves associated with performing open, freehand, and robotic-assisted laparoscopic pyeloplasty in a swine animal model. J Am Coll Surg 2009;208(4):576-586

7. Stefanidis D, Wang F, Korndorffer JRJr, Dunne JB, Scott DJ. Robotic assistance improves intracorporeal suturing performance and safety in the operating room while decreasing operator workload. Surg Endosc 2010;24(2):377-382

8. Lenihan JPJr, Kovanda C, Seshadri-Kreaden U. What is the learning curve for robotic assisted gynecologic surgery? J Minim Invasive Gynecol 2008;15(5):589-594

9. Seamon LG, Fowler JM, Richardson DL, et al. A detailed analysis of the learning curve: robotic hysterectomy and pelvicaortic lymphadenectomy for endometrial cancer. Gynecol Oncol 2009;114(2):162-167

10. Akl MN, Long JB, Giles DL, et al. Robotic-assisted sacrocolpopexy: technique and learning curve. Surg Endosc 2009;23(10):2390-2394

11. Ahmad G, Watson A, Vandekerckhove P, Lilford R. Techniques for pelvic surgery in subfertility. Cochrane Database Syst Rev 2006;(2):CD000221

12. Degueldre M, Vandromme J, Huong PT, Cadière GB. Robotically assisted laparoscopic microsurgical tubal reanastomosis: a feasibility study. Fertil Steril 2000;74(5):1020-1023

13. Rodgers AK, Goldberg JM, Hammel JP, Falcone T. Tubal anastomosis by robotic compared with outpatient minilaparotomy. Obstet Gynecol 2007;109(6):1375-1380

14. Dharia Patel SP, Steinkampf MP, Whitten SJ, Malizia BA. Robotic tubal anastomosis: surgical technique and cost effectiveness. Fertil Steril 2008;90(4):1175-1179

15. Mais V, Ajossa S, Guerriero S, Mascia M, Solla E, Melis GB. Laparoscopic versus abdominal myomectomy: a prospective, randomized trial to evaluate benefits in early outcome. Am J Obstet Gynecol 1996;174(2):654-658

16. Seracchioli R, Rossi S, Govoni F, et al. Fertility and obstetric outcome after laparoscopic myomectomy of large myomata: a randomized comparison with abdominal myomectomy. Hum Reprod 2000;15(12):2663-2668

17. Palomba S, Zupi E, Falbo A, et al. A multicenter randomized, controlled study comparing laparoscopic versus minilaparotomic myomectomy: reproductive outcomes. Fertil Steril 2007;88(4):933-941
18. Seracchioli R, Manuzzi L, Vianello F, et al. Obstetric and delivery outcome of pregnancies achieved after laparoscopic myomectomy. Fertil Steril 2006;86(1):159-165

19. Sizzi O, Rossetti A, Malzoni M, et al. Italian multicenter study on complications of laparoscopic myomectomy. J Minim Invasive Gynecol 2007;14(4):453-462

20. Liu G, Zolis L, Kung R, Melchior M, Singh S, Cook EF. The laparoscopic myomectomy: a survey of Canadian gynaecologists. J Obstet Gynaecol Can 2010;32(2):139-148

21. Advincula AP, Song A, Burke W, Reynolds RK. Preliminary experience with robot-assisted laparoscopic myomectomy. J Am Assoc Gynecol Laparosc 2004;11(4):511-518

22. Koh C, Janik G. Laparoscopic myomectomy: the current status. Curr Opin Obstet Gynecol 2003;15(4):295-301

23. Bedient CE, Magrina JF, Noble BN, Kho RM. Comparison of robotic and laparoscopic myomectomy. Am J Obstet Gynecol 2009;201(6):566; e1-e5

24. Greenberg JA, Einarsson JI. The use of bidirectional barbed suture in laparoscopic myomectomy and total laparoscopic hysterectomy. J Minim Invasive Gynecol 2008;15(5):621-623

25. Rackow BW, Arici A. Fibroids and in-vitro fertilization: which comes first? Curr Opin Obstet Gynecol 2005;17(3): 225-231

26. Moghadam R, Lathi RB, Shahmohamady B, et al. Predictive value of magnetic resonance imaging in differentiating between leiomyoma and adenomyosis. JSLS 2006;10(2): 216-219

27. Meredith SM, Sanchez-Ramos L, Kaunitz AM. Diagnostic accuracy of transvaginal sonography for the diagnosis of adenomyosis: systematic review and metaanalysis. Am J Obstet Gynecol 2009;201(1):107; e1-e6

28. Goto A, Takeuchi S, Sugimura K, Maruo T. Usefulness of Gd-DTPA contrast-enhanced dynamic MRI and serum determination of $\mathrm{LDH}$ and its isozymes in the differential diagnosis of leiomyosarcoma from degenerated leiomyoma of the uterus. Int J Gynecol Cancer 2002;12(4):354-361

29. Nezhat C, Lavie O, Hsu S, Watson J, Barnett O, Lemyre M. Robotic-assisted laparoscopic myomectomy compared with standard laparoscopic myomectomy-a retrospective matched control study. Fertil Steril 2009;91(2):556-559

30. De Hondt A, Meuleman C, Tomassetti C, Peeraer K, D'Hooghe TM. Endometriosis and assisted reproduction: the role for reproductive surgery? Curr Opin Obstet Gynecol 2006;18(4):374-379

31. Coccia ME, Rizzello F, Cammilli F, Bracco GL, Scarselli G. Endometriosis and infertility Surgery and ART: An integrated approach for successful management. Eur J Obstet Gynecol Reprod Biol 2008;138(1):54-59

32. Nezhat C, Lewis M, Kotikela S, et al. Robotic vs. standard laparoscopy for the treatment of endometriosis. Fertil Steril 2010;94(7):2758-2760

33. Lee G, Lee T, Dexter D, Klein R, Park A. Methodological infrastructure in surgical ergonomics: a review of tasks, models, and measurement systems. Surg Innov 2007;14(3): 153-167

34. Wiegmann DA, ElBardissi AW, Dearani JA, Daly RC, Sundt TMIII. Disruptions in surgical flow and their relationship to surgical errors: an exploratory investigation. Surgery 2007;142(5):658-665

35. Sutton E, Youssef Y, Meenaghan N, et al. Gaze disruptions experienced by the laparoscopic operating surgeon. Surg Endosc 2010;24(6):1240-1244 
36. Park A, Lee G, Seagull FJ, Meenaghan N, Dexter D. Patients benefit while surgeons suffer: an impending epidemic. J Am Coll Surg 2010;210(3):306-313

37. Lee G, Lee T, Dexter D, et al. Ergonomic risk associated with assisting in minimally invasive surgery. Surg Endosc 2009;23(1):182-188

38. Horgan S, Cullen JP, Talamini MA, et al. Natural orifice surgery: initial clinical experience. Surg Endosc 2009;23(7): $1512-1518$

39. Kalloo AN, Singh VK, Jagannath SB, et al. Flexible transgastric peritoneoscopy: a novel approach to diagnostic and therapeutic interventions in the peritoneal cavity. Gastrointest Endosc 2004;60(1):114-117

40. Rattner D, Kalloo A; ASGE/SAGES Working Group. ASGE/SAGES Working Group on Natural Orifice Translumenal Endoscopic Surgery. October 2005. Surg Endosc 2006;20(2):329-333

41. Hazey JW, Narula VK, Renton DB, et al. Natural-orifice transgastric endoscopic peritoneoscopy in humans: Initial clinical trial. Surg Endosc 2008;22(1):16-20

42. Steele K, Schweitzer MA, Lyn-Sue J, Kantsevoy SV. Flexible transgastric peritoneoscopy and liver biopsy: a feasibility study in human beings (with videos). Gastrointest Endosc 2008;68(1):61-66

43. Nezhat CH, Datta MS, Defazio A, Nezhat F, Nezhat C. Natural orifice-assisted laparoscopic appendectomy. JSLS 2009;13(1):14-18

44. Bergström M, Swain P, Park PO. Measurements of intraperitoneal pressure and the development of a feedback control valve for regulating pressure during flexible transgastric surgery (NOTES). Gastrointest Endosc 2007;66(1):174-178

45. Suzuki S, Suzuki N, Hattori A, Otake Y, Hashizume M. Telecontrol function of an endoscopic surgical robot with two hands for tele-NOTES surgery. Stud Health Technol Inform 2008;132:511-513

46. Thele F, Zygmunt M, Glitsch A, Heidecke CD, Schreiber A. How do gynecologists feel about transvaginal NOTES surgery? Endoscopy 2008;40(7):576-580

47. Hernandez JM, Morton CA, Ross S, Albrink M, Rosemurgy AS. Laparoendoscopic single site cholecystectomy: the first 100 patients. Am Surg 2009;75(8):681-685; discussion 685-686
48. Dutta S. Early experience with single incision laparoscopic surgery: eliminating the scar from abdominal operations. J Pediatr Surg 2009;44(9):1741-1745

49. Tacchino RM, Greco F, Matera D. Laparoscopic gastric banding without visible scar: a short series with intraumbilical SILS. Obes Surg 2010;20(2):236-239

50. Rao PP, Bhagwat SM, Rane A, Rao PP. The feasibility of single port laparoscopic cholecystectomy: a pilot study of 20 cases. HPB (Oxford) 2008;10(5):336-340

51. Savaris RF, Cavazzola LT. Ectopic pregnancy: laparoendoscopic single-site surgery-laparoscopic surgery through a single cutaneous incision. Fertil Steril 2009;92(3):1170; e5-e7

52. Yoon BS, Park H, Seong SJ, Park CT, Park SW, Lee KJ. Single-port laparoscopic salpingectomy for the surgical treatment of ectopic pregnancy. J Minim Invasive Gynecol 2010;17(1):26-29

53. Fader AN, Escobar PF. Laparoendoscopic single-site surgery (LESS) in gynecologic oncology: technique and initial report. Gynecol Oncol 2009;114(2):157-161

54. Ostrowitz MB, Eschete D, Zemon H, DeNoto G. Roboticassisted single-incision right colectomy: early experience. Int J Med Robot 2009;5(4):465-470

55. Ishikawa N, Arano Y, Shimizu S, et al. Single incision laparoscopic surgery (SILS) using cross hand technique. Minim Invasive Ther Allied Technol 2009;18(6):322324

56. Tacchino R, Greco F, Matera D. Single-incision laparoscopic cholecystectomy: surgery without a visible scar. Surg Endosc 2009;23(4):896-899

57. Saber AA, El-Ghazaly TH. Early experience with singleaccess transumbilical adjustable laparoscopic gastric banding. Obes Surg 2009;19(10):1442-1446

58. Philipp SR, Miedema BW, Thaler K. Single-incision laparoscopic cholecystectomy using conventional instruments: early experience in comparison with the gold standard. J Am Coll Surg 2009;209(5):632-637

59. Chamberlain RS, Sakpal SV. A comprehensive review of single-incision laparoscopic surgery (SILS) and natural orifice transluminal endoscopic surgery (NOTES) techniques for cholecystectomy. J Gastrointest Surg 2009;13(9): 1733-1740 